\title{
New Exploration of Packet-Pair Probing for Available Bandwidth Estimation and Traffic Characterization
}

\author{
Yu Cheng*, Vikram Ravindran ${ }^{\dagger}$, Alberto Leon-Garcia ${ }^{\dagger}$, Hsiao-Hwa Chen $^{\ddagger}$ \\ ${ }^{*}$ Department of Electrical and Computer Engineering, Illinois Institute of Technology, Chicago, IL, USA \\ Email: cheng@iit.edu \\ ${ }^{\dagger}$ Department of Electrical and Computer Engineering, University of Toronto, Toronto, ON, Canada \\ Emails: \{v.ravindran, alberto.leongarcia\}@utoronto.ca \\ ${ }^{\ddagger}$ Institute of Communications Engineering, National Sun Yat-Sen University, Kaohsiung City, Taiwan \\ Emails: hshwchen@mail.nsysu.edu.tw
}

\begin{abstract}
The Packet-pair dispersion techniques are the most common probing-based approach to measuring the bottleneck capacity of a path. In practice, the dispersion measurement, and therefore the bandwidth estimation, could be seriously distorted by the cross traffic queuing between or in front of the probe packet pair. Almost all the existing packet-pair techniques depend on heuristic filtering methods to find a final capacity estimate. In this paper, we take a different perspective to exploit the crosstraffic effect. We develop a queueing model to describe the output packet-pair dispersions interfered by the cross traffic, based on which a new measurement technique to estimate the available bandwidth is derived. Another important contribution is that we for the first time reveal that the statistics of the cross traffic, e.g. the marginal distribution and the autocovariance function of the arrival process, can also be inferred from the stochastic behavior of the output packet dispersions. Efficiency of the proposed available bandwidth estimation and traffic characterization techniques are demonstrated by computer simulations.
\end{abstract}

\section{INTRODUCTION}

The end-based network measurement has attracted wide attention in recent years, by which internal network status is indirectly inferred from end-to-end or edge-to-edge active or passive traffic measurements [1]. The end-based approach is more feasible than the router-based approach by requiring cooperation of only the path end-points. While the end-based approach greatly facilitates customers' awareness of network status, it can also bring higher flexibility and scalability to the network management [2], [3].

Packet dispersion techniques [4]-[7] are the most popular end-based approach for bandwidth measurement. Two bandwidth metrics that are commonly associated with a path are the capacity and the available bandwidth [7]. According to [7], we refer to the capacity limiting link as narrow link and to the available bandwidth limiting link as tight link, to avoid the ambiguity of the term bottleneck link which has been widely used for both metrics. Among the packet dispersion techniques, the pack-pair dispersion techniques are normally used for measuring the capacity of a path, and the packet-train dispersion techniques normally for measuring the available bandwidth of a path [8]. This paper focuses on the packetpair techniques.

The basic idea of the packet-pair technique is that when a back-to-back probe packet pair of the same size is launched into a network, assuming no cross-traffic, the packet pair will be maximally dispersed at the narrow link, and the dispersion will go through the downstream links to reach the destination. If knowing the probe packet size, the receiver can then estimate the capacity of the path from the measured dispersion. Unfortunately, the cross traffic, referring to the Internet traffic that shares the same first-come-first-serve (FCFS) buffer with the probe packet pair, exist in practice. The dispersion received can be either expanded by the cross-traffic packets queueing between the probe packet pair at the narrow link, or compressed by the cross-traffic packets queueing in front of the probe pair downstream of the narrow link. The expansion or compression of the dispersion will lead to under-estimation or over-estimation of the path capacity, respectively. To overcome this problem, the common approach is to generate a reasonable number of dispersion samples, maybe from packet pairs with different sizes, and use some heuristic filtering methods to find the final bandwidth estimation [5], [6], [9], [10].

In this paper, we give a new exploitation of the packetpair dispersion techniques by developing a queueing model to describe the impact of the cross traffic on the packet-pair dispersion. The study is motivated by the objective to build a systematic methodology to infer internal network status from end-to-end measurements. Based on the queueing model, we find that the queueing behavior of the probe packet pair can be divided into two situations. One is that the packet pair are served in the same busy period of the queue, where the packet pair is termed as a united pair; the other is that the two probe packets fall into different busy periods, where the packet pair is termed as a divided pair. The dispersions from the united pairs and divided pairs have different distributions. While the divided-pair dispersions are exploited to derive a simple and efficient available bandwidth measurement approach, the united-pair dispersions are exploited for traffic characteri- 
zation, that is, extracting the statistics (e.g. the marginal distribution and the autocovariance function [11], [12]) of the cross traffic. It is noteworthy that traffic characterization can be used to enhance QoS control on the customer side and facilitate resource management on the network side through queueing analysis or effective bandwidth techniques [12]-[14]. To the best of our knowledge, no similar dispersion technique for traffic characterization has been presented in the literature.

The remainder of this paper is organized as follows. Section II gives a review of the related work. Section III describes the queueing model governing the output dispersions under the cross traffic. Section IV and Section V present the proposed available bandwidth measurement and the traffic characterization techniques, respectively. Section VI presents simulation results to demonstrate the performance. Section VII gives the concluding remarks.

\section{RELATED WORK}

It is indicated in [15] that the dispersion expansion or compression due to the cross-traffic leads to multimodal distribution of the bandwidth measurements. The relationship between the network load, cross-traffic packet size, probe packet size and the location and strength of different modes has been investigated in [7], based on which a filtering methodology is proposed. Pasztor and Veitch proposed a queueing model in [16] to formalize and unify the network dispersion effect, which is the most related work to our study. Nevertheless, we step further in this paper to decode the statistic information of the cross traffic from the dispersion measurements. Moreover, in [16] the available bandwidth is estimated by united-pair measurement (according to our terminologies), while in this paper we estimate the available bandwidth by divided-pair measurement.

With FCFS forwarding, the available bandwidth is only meaningful over an averaging timescale [8]. Therefore, the packet-train dispersion techniques [6], [17], instead of the packet-pair dispersion, are normally used to measure the available bandwidth. Recently, Dovrolis, Ramanathan, and Moore showed that the dispersion of long packet trains does not measure the available bandwidth of a path; instead, it measures a different throughput metric referred to as the asymptotic dispersion rate (ADR) [7], [18]. The SLoPS technique [8] detects the available bandwidth by sending probe packet streams with variable testing rates, based on the observation that the one-way delay of a packet stream increase obviously when the streams' rate is higher than the available bandwidth. The TOPP technique [19] shows that such a delay principle can be combined with the packet-pair dispersion measurement to estimate the available bandwidth. Note that the available bandwidth estimation techniques proposed in this paper also use packet-pair dispersion measurement, but based on queueing analysis.

\section{PACKET-PAIR DISPERSION}

In this section, we describe a queueing model to formalize the packet-pair dispersion behavior. We model a store-and-

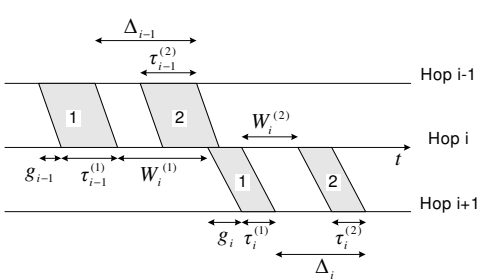

(a)

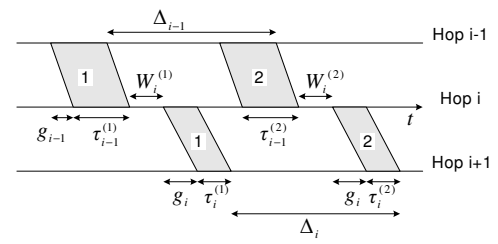

(b)

Fig. 1. Two types of dispersion behaviors: (a) the second packet reaches queue $i$ before the first leaves, (b) the second packet reaches queue $i$ after the first has departed.

forward router on a path as a FCFS queue which is served at a fixed rate of $C$ bits per second (or equivalently bytes per second), the capacity of the link connected to the router. A network path consisting of links from 1 to $n$ is modeled as the concatenation of $n$ queues, where each queue has serving capacity $C_{i}(1 \leq i \leq n)$. We define a probe packet pair as two consecutive probe packets that are transmitted along a path between the source and the destination. The first packet in the pair has a size $L^{(1)}$ bits, and the second packet has a size $L^{(2)}$. If $L^{(1)}=L^{(2)}$, then the packet-pair is called symmetric, otherwise it is called asymmetric. We refer to other packets in the network other than the probe traffic as cross traffic.

\section{A. Dispersion Governing Equations}

The packet-pair dispersion at a given point is defined as the the time when the last bit of the first packet arrives at the point to the time when the last bit of the second packet arrives. The dispersion of a packet pair leaving queue $i$ is denoted as $\Delta_{i}$, also termed as the output dispersion of queue $i$. We assume that the propagation delay along a link is constant, and therefore the output dispersion from a queue is equal to the input dispersion at the next downstream queue.

The packet-pair dispersion behavior at a queue $i$ can fall in two cases [7], as illustrated in Fig. 1. In one case the second probe packet arrives before the first probe packet leaves the queue, and in the other case the second probe packet arrives after the first probe packet has left. For queue $i$, the two cases of the output dispersion are described by the following governing equations [7]:

$$
\Delta_{i}= \begin{cases}\sigma_{i}^{(2)} & \text { if } \Delta_{i-1} \leq \sigma_{i}^{(1)} \\ \Delta_{i-1}+\sigma_{i}^{(2)}-\sigma_{i}^{(1)} & \text { otherwise }\end{cases}
$$

where $\sigma_{i}^{(j)}=\tau_{i}^{(j)}+W_{i}^{(j)}$ and $\tau_{i}^{(j)}=\frac{L^{(j)}}{C_{i}}$ for $j=1,2 . W_{i}^{(1)}$ is the queueing delay of the first probe packet, and $W_{i}^{(2)}$ is the queueing delay of the second probe packet due to the cross traffic inserted between the probe packet pair. 


\section{B. Cross-Traffic Effect}

In the dispersion governing equations, the cross traffic affects the output dispersion through the queueing delay $W_{i}^{(j)}$. To explicitly describe the cross traffic effect, we classify the packet-pair probing into two cases. The first case is defined as united-pair probing, where the two probe packets fall in the same busy period of the queueing process. In this case, the server is always busy before the second packet arrives, while the first probe packet may depart before or after the second probe packet arrives. Let $A(t)$ denote the cross-traffic arrival process (in bytes) to the queue, the output dispersion in this case is

$$
\Delta_{i}=\frac{A\left(\Delta_{i-1}\right)+L^{(2)}}{C_{i}} .
$$

The second case is defined as divided-pair probing, where the two probe packets fall in different busy periods of the queueing process. In this case, the first probe packet definitely leaves before the second probe packet arrives, as shown in Fig. 1(b). The output dispersion in this case is

$$
\Delta_{i}=\Delta_{i-1}+\frac{L^{(2)}-L^{(1)}}{C_{i}}+W_{i}^{(2)}-W_{i}^{(1)} .
$$

In summary, the output dispersion under the cross-traffic effect can be expressed as

$\Delta_{i}=\left\{\begin{array}{l}\frac{A\left(\Delta_{i-1}\right)}{C_{i}}+\frac{L^{(2)}}{C_{i}} \\ \Delta_{i-1}+\frac{L^{(2)}-L^{(1)}}{C_{i}}+W_{i}^{(2)}-W_{i}^{(1)}\end{array}\right.$

\section{Available Bandwidth Estimation}

According to the dispersion governing equation (4), the united-pair measurement is normally combined with the existing filtering techniques to estimate the capacity of the narrow link along a path [7]. In this section, we propose a novel divided-pair measurement method derived from (4) to estimate the available bandwidth of a path.

The available bandwidth is estimated through the utilization measurement. In a FCFS work-conserving queue, the utilization factor $u$ is defined as the proportion of time that the link is busy. Assume the queue is infinite and no packet loss happens, the utilization of link- $i$ can be expressed as

$$
u_{i}=\frac{r_{i}}{C_{i}}
$$

where $C_{i}$ is the link capacity, and $r_{i}=\lim _{t \rightarrow \infty} \frac{A_{i}(t)}{t}$ is the average rate of the traffic arrival process $A_{i}(t)$ to queue- $i$. According to [7], the available bandwidth at link $i$ is defined as $A_{i}=C_{i}\left(1-u_{i}\right)$, and the available bandwidth of a path having $H$ hops is

$$
A=\min _{i=1 \ldots H} C_{i}\left(1-u_{i}\right)=A_{\text {tight-link }} .
$$

With symmetric united-pair probing, it is not difficult to derive from (4) that

$$
u_{i}=\frac{E\left[\Delta_{i}\right]-L^{(2)} / C_{i}}{\Delta_{i-1}} .
$$

In this method, the link capacity should be measured first for utilization estimation; the capacity estimation error will then impact the utilization measurement. Such a disadvantage can be avoided by divided-pair measurement.

If we use symmetric divided-pairs, from (4), we know that

$$
\Delta_{i}=\Delta_{i-1}+W_{i}^{(2)}-W_{i}^{(1)}
$$

As the two probe packets are in different busy periods, the amount of queued traffic they see (and therefore the two delaytimes $W_{i}^{(1)}$ and $W_{i}^{(2)}$ ) are independent and have the same distribution, assuming a stationary queueing process.

In order to find the utilization, we first investigate the probability of $W_{i}^{(1)}=W_{i}^{(2)}$. Considering the possible two cases, we have

$$
\begin{aligned}
P\left[W_{i}^{(1)}=W_{i}^{(2)}\right]= & P\left[W_{i}^{(1)}=W_{i}^{(2)}=0\right]+ \\
& P\left[W_{i}^{(1)}=W_{i}^{(2)} \neq 0\right] .
\end{aligned}
$$

It is far more likely for the waiting times to be equal due to that both probe packets found the queue empty (a common occurrence, especially in low-utilization cases) than due to that the two non-zero waiting times are equal. Therefore, it is reasonable to get the approximation

$$
\begin{aligned}
P\left[W_{i}^{(1)}=W_{i}^{(2)}\right] & \approx P\left[W_{i}^{(1)}=0, W_{i}^{(2)}=0\right] \\
& =P\left[W_{i}^{(1)}=0\right] P\left[W_{i}^{(2)}=0\right] .
\end{aligned}
$$

Furthermore, according to the definition of the utilization factor, we have ${ }^{1}$,

$$
P\left[W_{i}^{(1)}=0\right]=P\left[W_{i}^{(2)}=0\right] \approx P[\text { link is idle }]=1-u_{i} .
$$

Combining (7), (8), and (9), we obtain

$$
P\left[\Delta_{i}=\Delta_{i-1}\right]=P\left[W_{i}^{(1)}=W_{i}^{(2)}\right] \approx\left(1-u_{i}\right)^{2}
$$

where an estimator of $u_{i}$ can then be derived as

$$
\hat{u}_{i} \approx 1-\sqrt{P\left[\Delta_{i}=\Delta_{i-1}\right]} .
$$

It is expected that the estimator of (11) has a good performance when the utilization is not very high, which will be demonstrated through simulations in Section VI. The estimator can also be applied to a multi-queue path. Without loss of generality, we denote link-1 as the tight link of a path with $H$ hops. The relationship of (10) can then be extended to

$$
P\left[\Delta_{i}=\Delta_{i-1}\right] \approx\left(1-u_{1}\right)^{2} \prod_{i=2}^{H}\left(1-u_{i}\right)^{2} .
$$

If the utilization of other links is much lower than that of the tight link, i.e. $\prod_{i=2}^{H}\left(1-u_{i}\right)^{2} \approx 1$, the estimator of (11) can then well approximate $u_{1}$. In a more strict sense, it is not difficult to see that the estimation from (11) gives a upper bound of $u_{1}$ in the multi-queue case. To the best our knowledge, no measurement technique similar to the proposed divided-pair estimation has yet been presented in the literature.

\footnotetext{
${ }^{1}$ If the input process is a Poisson process, the “ $\approx$ " in $(9)$ can be replaced by a "=", according to the PASTA theory (i.e. Poisson arrivals see time average).
} 


\section{TRAFFIC CHARACTERIZATION}

In this section, we show that the packet-pair dispersion measurements can be used to estimate the statistical characteristics, e.g. the marginal distribution and the autocovariance function of the cross-traffic packet arrival process. To facilitate the estimation, we use the independent and identically distributed (iid) packet-size assumption and the fact that Internet packet size follows a known multi-modal distribution.

\section{A. Internet Packet Size Distribution}

Internet measurements have shown that packets of a few sizes tend to make up the vast majority of Internet packets [20]-[23]. As an example, we refer to the measurements performed by the Cooperative Association for Internet Data Analysis (CAIDA) on the traffic passing through the NASA Ames Internet Exchange (AIX) between February 21st and 27 th, 2000 [20]. The cumulative distribution function of the packet sizes is re-plotted in Fig. 2. From the figure, it can be seen that the vast majority of packets were of a few sizes, i.e. 40 bytes, 576 bytes, and 1500 bytes, which is attributed to the behavior of the Transport Control Protocol (TCP). As CAIDA does not provide the histogram, we produce an approximate numerical probability mass function (PMF) from Fig. 2:

$$
P[S=k]= \begin{cases}0 & k \in\{0,1, \ldots, 39\} \\ 0.50 & k=40 \\ \frac{0.13}{575-40} & k \in\{41,42, \ldots, 575\} \\ 0.17 & k=576 \\ \frac{0.03}{1499-576} & k \in\{577, \ldots, 1499\} \\ 0.17 & k=1500\end{cases}
$$

\section{B. Cross-Traffic Marginal Distribution Estimation}

In the following, we develop a method to estimate the marginal distribution of packet arrival process and the marginal packet-size distribution, based on the probability generating function (PGF).

Consider a united-pair with dispersion $\Delta$ is sent to probe a queue. Cross-traffic can be completely described by two random variables, which are the random variable $N(\Delta)$ to denote the number of packets that arrive in a given interval $\Delta$, and the random variable $S$ to denote the packet size. The total amount of cross-traffic $A(\Delta)$ can then be expressed as $A(\Delta)=\sum_{n=0}^{N(\Delta)} S_{n}$, where $S_{n}$ are iid random variables.

Let us define the PGFs of $N(\Delta)$ and $X$ as

$$
\begin{gathered}
G_{N(\Delta)}(z)=E\left[z^{N(\Delta)}\right]=\sum_{k=0}^{\infty} P[N(\Delta)=k] z^{k} \\
G_{S}(z)=E\left[z^{S}\right]=\sum_{k=0}^{\infty} P[S=k] z^{k} .
\end{gathered}
$$

According to the independence assumptions given at the beginning of this Section, it is the well-known result [24] that

$$
\begin{aligned}
G_{A(\Delta)}(z) & =E\left[z^{A(\Delta)}\right]=E\left[E\left[z^{A(\Delta)} \mid N(\Delta)\right]\right] \\
& =G_{N(\Delta)}\left(G_{S}(z)\right) .
\end{aligned}
$$

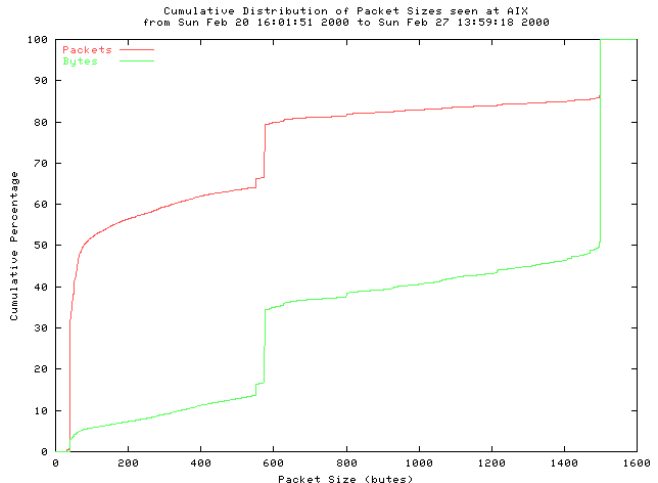

Fig. 2. Packet size distribution at NASA AIX, February 21-27,2000

With united-pair measurement, the total cross-traffic volume can be obtained from the dispersions. Again, using $\Delta_{i-1}$ and $\Delta_{i}$ to represent the input and output dispersions associated with the queue under consideration, respectively, we have

$$
A\left(\Delta_{i-1}\right)=C_{i} \Delta_{i}-L^{(2)}
$$

By sending a reasonable number of probe packet pairs, the histogram of $A(\Delta)$ (we drop the subscription of $\Delta_{i-1}$ for the convenience of expression), and therefore the corresponding PGF $G_{A(\Delta)}(z)$, can be calculated. Using $q_{k}(\Delta)$ to denote the histogram probability $P[A(\Delta)=k]$, the PGF $G_{A(\Delta)}(z)$ is then calculated as

$$
G_{A(\Delta)}(z)=\sum_{k=0}^{\infty} q_{k}(\Delta) z^{k} .
$$

Given $G_{A(\Delta)}(z)$, our objective is to estimate the marginal distribution $P[N(\Delta)=n]$, briefly denoted as $p_{n}(\Delta)$, for $n=0,1, \ldots, \infty$.

One fact we are to use is that the Internet packet size distribution takes only a few, say $m$, dominant values as we have discussed. Then, $G_{S}(z)$ reduces to

$$
G_{S}(z)=a_{1} z^{l_{1}}+a_{2} z^{l_{2}}+\cdots+a_{m} z^{l_{m}}
$$

where $l_{1}, l_{2}, \ldots, l_{m}$, arranged as $l_{1}<l_{2}, \cdots<l_{m}$, are the $m$ dominant packet sizes and $a_{1}, a_{2}, \ldots, a_{m}$ are the corresponding probabilities. Define the probability vector $\boldsymbol{p}=$ $\left(p_{0}, p_{1}, \ldots, p_{l_{m}}\right)$, where $p_{l_{k}}=a_{k}(k=1, \ldots m)$ and other values in the vector are equal to 0 . If use $\boldsymbol{p}^{(n)}=$ $\left(p_{0}^{(n)}, p_{1}^{(n)}, \ldots, p_{n l_{m}}^{(n)}\right)$ to denote the probability vector of the random variable $S^{(n)}=\sum_{i=1}^{n} S_{i}$, and $\otimes$ the convolution operation, we have

$$
\boldsymbol{p}^{(n)}=\boldsymbol{p} \otimes \boldsymbol{p} \cdots \otimes \boldsymbol{p}(\text { with } n \text { fold }) .
$$

For the PGF, we have

$$
G_{S^{(n)}}(z)=\left(G_{S}(z)\right)^{n}=\sum_{k=0}^{n l_{m}} p_{k}^{(n)} z^{k} .
$$


Based on the result of (21), (16) can be unwrapped as

$$
\begin{aligned}
G_{A(\Delta)}(z) & =G_{N(\Delta)}\left(G_{S}(z)\right)=\sum_{n=0}^{\infty}\left(G_{S}(z)\right)^{n} p_{n}(\Delta) \\
& =\sum_{n=0}^{\infty} \sum_{k=0}^{n l_{m}} p_{k}^{(n)} z^{k} p_{n}(\Delta)=\sum_{k=0}^{\infty}\left[\sum_{n=0}^{\infty} p_{k}^{(n)} p_{n}(\Delta)\right] z^{k} .
\end{aligned}
$$

Comparing (18) and (22), we can get

$$
q_{k}(\Delta)=\sum_{n=0}^{\infty} p_{k}^{(n)} p_{n}(\Delta) .
$$

It is possible to solve $p_{n}(\Delta)$ from (23). However, it is impractical to estimate $p_{n}(\Delta)$ up to $n \rightarrow \infty$. In practice, the cross-traffic input to a queue is limited by the upstream link capacity. Letting $L_{\text {min }}$ denote the minimum packet size allowed, we can obtain an upper bound of $N(\Delta)$ as

$$
N(\Delta) \leq N_{\max }^{\Delta}=C_{i-1} \Delta / L_{\min }
$$

and (23) reduces to

$$
q_{k}(\Delta)=\sum_{n=0}^{N_{\max }^{\Delta}} p_{k}^{(n)} p_{n}(\Delta) .
$$

With vectors $\boldsymbol{p}^{(n)}\left(n=0,1, \ldots, N_{\max }^{\Delta}\right)$ available from (20), define $\boldsymbol{p}_{(k)}=\left(p_{k}^{(0)}, p_{k}^{(1)}, \ldots, p_{k}^{\left(N_{\text {max }}^{\Delta}\right)}\right)$. Assume that we can find $N_{\max }^{\Delta}+1$ points of $k$, i.e. $k_{0}, k_{1}, \ldots, k_{N_{\max }^{\Delta}}$, so that the $\left(N_{\max }^{\Delta}+1\right) \times\left(N_{\max }^{\Delta}+1\right)$ matrix $\boldsymbol{P}$, with $\boldsymbol{p}_{\left(k_{i}\right)}$ for $i=0,1, \ldots, N_{\max }(\Delta)$ as the row vectors, is invertible. Moreover, from the histogram of $A(\Delta)$, we can have the values of $\left(q_{k_{0}}(\Delta), q_{k_{1}}(\Delta), \ldots, q_{k_{N_{\Delta}}}(\Delta)\right)$, denoted as vector $\boldsymbol{q}(\Delta)$. Use the vector $\boldsymbol{p}(\Delta)$ to denote the cross-traffic marginal distribution $\left(p_{0}(\Delta), p_{1}(\Delta), \ldots, p_{N_{\max }^{\Delta}}(\Delta)\right)$, which can be estimated as

$$
\boldsymbol{p}(\Delta)^{T}=\boldsymbol{P}^{-1} \boldsymbol{q}(\Delta)^{T} .
$$

In the above, we have discussed the cross-traffic marginal distribution estimation in the single-queue context. In a multiqueue path, if there is only one bottleneck, narrow as well as tight, and the downstream links only incur ignorable delay, the estimation of (26) is expected to have an acceptable performance.

\section{Packet Size Distribution Estimation}

We can also estimate the packet size distribution based on (16), if the packet arrival marginal distribution is available from traffic modeling or from historical data measurements. The estimation is straightforward as

$$
G_{S}(z)=G_{N(\Delta)}^{-1}\left(G_{A(\Delta)}(z)\right) \text {. }
$$

For example, let's assume that the self-similar Internet traffic can be well modeled by an $M / G / \infty$ input process [11]. The Marginal distribution is then Poisson with average rate $\lambda$ packets/second, giving $G_{N(\Delta)}=e^{\lambda \Delta(z-1)}$. Using this result,

$$
G_{S}(z)=1+\frac{1}{\lambda \Delta} \ln \left(G_{A(\Delta)}(z)\right)
$$

With the PGF known, the probability vector $\boldsymbol{p}$ can be obtained with an inverse fast Fourier transform (IFFT) operation.

\section{Performance Evaluation}

In this section, we present some computer simulation results to illustrate the performance of the proposed traffic characterization techniques. We develop a single-queue simulator using the event-driven simulation technique in the MATLAB language. In all the simulation examples, we use the iid packetsize assumption, and the cross-traffic packet size distribution follows the CAIDA PMF given in (13).

\section{A. Utilization estimation}

In this example, we examine the performance of the utilization estimation technique based on divided-pair measurement. We simulate a $10 \mathrm{Mbps}$ queue. The packet-pairs are symmetric with size 1500 bytes, and inter-pair interval is $0.05 \mathrm{~s}$. Poisson cross traffic is sent for four different target utilizations $(0.10$, $0.30,0.50$, and 0.70 ) and five different input dispersions (0.0016, 0.0032, 0.0064, 0.0128 and 0.00256) second, to examine the goodness of the utilization estimator given in (11) over a wide range of scenarios. Each estimate is obtained from 10000 trials, and batch method is applied to find the $99 \%$ confidence interval.

The estimation results are given in Fig. 3(a)-3(d). Corresponding to each estimation point, there is a "actual utilization point", which is the accurate measurement of the true average utilization that occurs in the queue over the simulation run. The actual utilization is obtained by dividing the total time during which the queue is servicing a cross-traffic packet (probepacket service time is not counted) by the total time of the simulation run. From Fig. 3, we can see in all the utilization scenarios, the estimator has a robust performance where the actual utilization falls in the confident estimation range, except when input dispersion is too small, i.e. the case of 0.0016 seconds. There the estimator significantly overestimate the actual utilization. The reason is that the small input dispersion leads to united packet pairs, where the assumption that the waiting times of the two packets are independent breaks down, which contributes to the estimation deviation.

\section{B. Packet Size Distribution Estimation}

In this example, we illustrate the performance of the packet size distribution estimator given in (27). A 10 Mbps queue is simulated. We consider Poisson arrival process with packet size distribution following the CAIDA PMF. Four scenarios with the combination of $(u, \Delta)=(0.5,0.0006),(0.5,0.0012)$, $(0.99,0.0006)$, and $(0.99,0.0012)$, are simulated. Probe packet size is set correspondingly to guarantee the unite-pair measurement, and inter-pair interval is $0.05 \mathrm{~s}$. In all the scenarios, a series of 10000 probe pairs are sent for sampling, and $N_{\max }^{\Delta}=12$ is used considering that $\sum_{k=0}^{N_{\max }^{\Delta}} p_{k}(\Delta) \approx 1.0000$. The packet size distribution is estimated according to the following procedure.

- Measure the output dispersions and convert the dispersions to traffic loads in bytes according to (17). Generate 


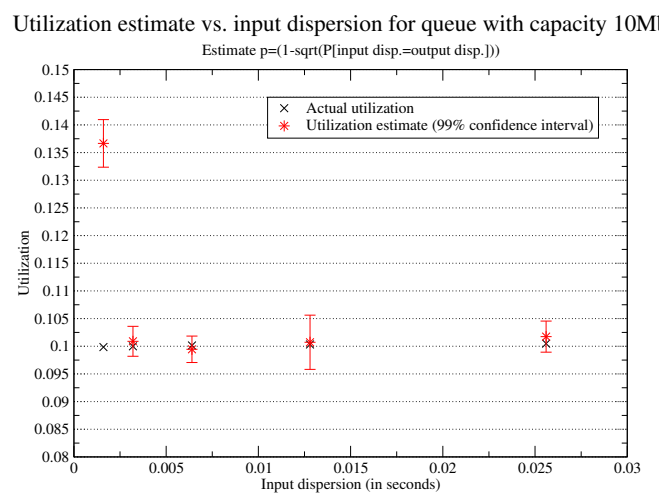

(a)

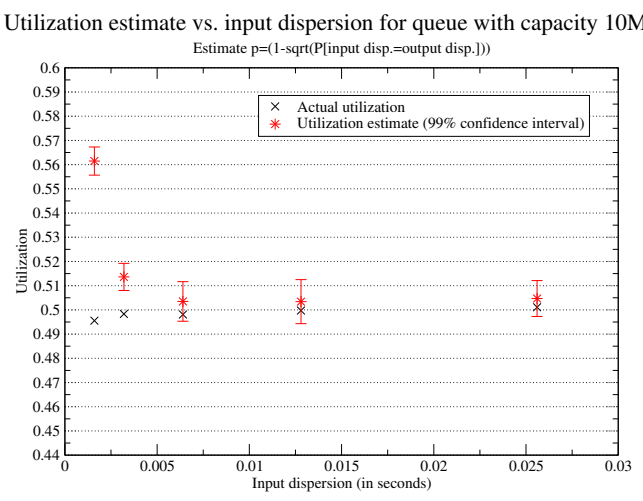

(c)

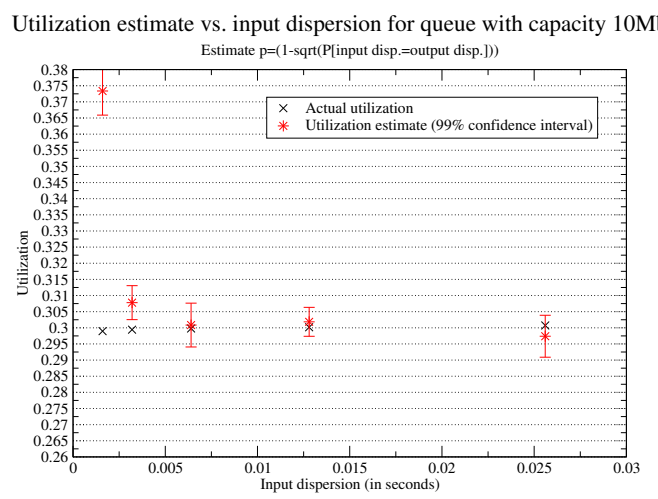

(b)

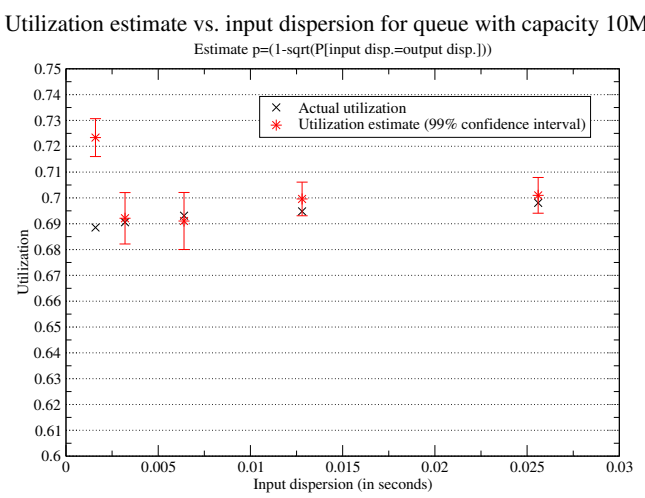

(d)

Fig. 3. Utilization estimation based on divided-pair measurement of a 10 Mbps queue with target utilization (a) 0.1 , (b) 0.3 , (c) 0.5 , and (d) 0.7 .

TABLE I

ESTIMATED CROSS-TRAFFIC PACKET SIZE DISTRIBUTION, USING IFFT METHOD

\begin{tabular}{c|c||c|c|c}
\hline \multicolumn{2}{c||}{ Scenario Simulated } & \multicolumn{3}{c}{ Estimated probabilities of three main modes } \\
\hline$u$ & $\Delta$ & 40 & 576 & 1500 \\
\hline \hline 0.50 & 0.0006 & 0.4854 & 0.1713 & 0.1744 \\
0.50 & 0.0012 & 0.5306 & 0.1737 & 0.1668 \\
0.99 & 0.0006 & 0.5192 & 0.1729 & 0.1747 \\
0.99 & 0.0012 & 0.4680 & 0.1497 & 0.1466 \\
\hline
\end{tabular}

the histogram of $A(\Delta)$, and then take the $\left(1500 N_{\text {max }}^{\Delta}+\right.$ 1)-point FFT of the histogram to obtain $G_{A}(z)$.

- Calculate $G_{S}(z)=1+\frac{1}{\lambda \Delta} \ln \left(G_{A}(z)\right)$.

- Take the $\left(1500 N_{\max }^{\Delta}+1\right)$-point IFFT of $G_{S}(z)$ to get the packet size distribution estimate $\hat{P}\{S=k\}$.

The estimated PMFs for the four scenarios are plotted in Figures 4(a) to 4(d). Although the maximum packet size is 1500 bytes, the FFT and IFFT operations will spread the estimation errors to all the points. In Fig. 4, $\hat{P}\{S=k\}$ for $k=0,1, \ldots, 3000$ are plotted to illustrate the error spreading. In all the four scenarios, the estimated distribution correctly identifies the three main packet sizes, i.e. 40, 576, and 1500 bytes, and the estimated probabilities are quite close to the true CAIDA values. The estimate values of the three dominant probabilities are presented in Table I.
In the figures, we can observe that estimation errors exist in all the scenarios and spread to points larger than 1500 . Also, the FFT and IFFT operations lead to negative estimates for some points. When the input dispersion or the utilization increases, the estimation error also increase. For example, the estimation in Fig. 4(d) with $(u, \Delta)=(0.99,0.0012)$ is much noiser than that in Fig. 4(a) with $(u, \Delta)=(0.5,0.0006)$. The estimation error is due to the insufficiency of measurement samples. In the case of $(u, \Delta)=(0.5,0.0006)$, the packet arrival rate is 0.8443 packets/dispersion, correspondingly, $\sum_{k=0}^{6} p_{k}(0.0006) \approx 1.0000$. In the case of $(u, \Delta)=(0.99,0.0012)$, the packet arrival rate is 3.3436 packets/dispersion, correspondingly, $\sum_{k=0}^{12} p_{k}(0.0012) \approx 1.0000$. With the same number of samples, many more modes need to be estimated in the latter case to obtain the histogram of $A(\Delta)$, therefore the accuracy decreases. We have done experiments to do estimation with more probing pairs, the noise in the case of $(u, \Delta)=(0.99,0.0012)$ can be obviously suppressed.

\section{Packet Arrival Process Characterization}

In this paper, we omit the performance evaluation of the marginal distribution estimation technique proposed in section V-B, due to the paper length limit. In stead, we give a detailed investigation in [25] of the traffic characterization technique using packet-pair probing. In [25], we discuss how to construct a invertible matrix $\boldsymbol{P}$ for marginal distribution 


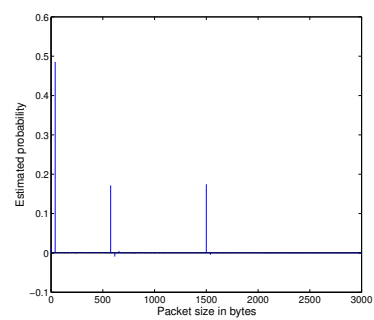

(a)

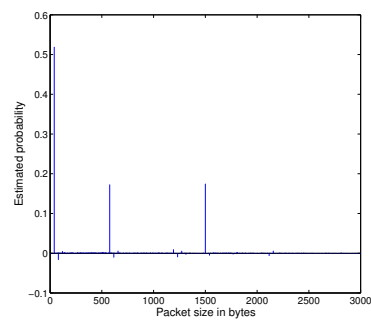

(c)

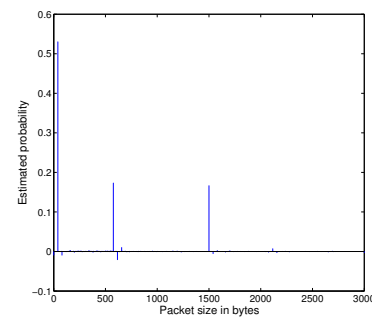

(b)

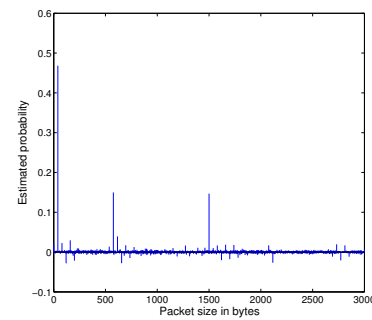

(d)
Fig. 4. Packet size distribution estimation in four scenarios, with $(u, \Delta)$ set as (a) $(0.5,0.0006),(b)(0.5,0.0012)$, (c) $(0.99,0.0006)$, and (d) $(0.99$, $0.0012)$.

estimation, extend the estimation technique to estimate the autocorrelation function of a self-similar process, and present simulation results to demonstrate the efficiency of the traffic characterization for both Poisson arrivals and self-similar arrivals. Moreover, we discuss how the edge-based traffic characterization is in fact a black box system that can be applied for end-to-end QoS provisioning along a path.

\section{CONCLUSIONS}

In this paper, we propose new packet-pair dispersion techniques for network measurement. As the dispersion measurement is taken at end systems or network edges, it considerably strengthen the customers' capability for QoS control and bring higher flexibility and scalability to network management. We develop a queueing model to describe the stochastic relationship among the input dispersion, the cross traffic, and the output dispersion. Based on the queuing model, new available bandwidth estimation and traffic characterization techniques have been derived. Particulary, traffic characterization, i.e. estimating the marginal distribution and autocovariance function of the traffic arrival process based on dispersion measurement, is a novel technique for the first time being presented. While bandwidth estimation mainly focus on the control of the average throughput, traffic characterization can facilitate the control of other QoS metrics, e.g. the delay and loss rate. Performance of the proposed bandwidth estimation and traffic characterization techniques have been demonstrated through computer simulations. We have discussed the proposed dispersion-techniques mainly for a single-queue or single-bottleneck path. Developing efficient multi-queue measurement techniques in a systematic way is still an open problem.

\section{REFERENCES}

[1] M. Coates, A. O. Hero III, R. Nowak, and B. Yu, "Internet tomography," IEEE Signal Processing Mag., vol. 19, pp. 47-65, May 2002.

[2] G. Bianchi, N. Blefari-Melazzi, M. Femminella, and F. Pugini, "Performance evaluation of a measurement-based algorithm for distributed admission control in a DiffServ framework," in Proc. IEEE GLOBECOM'01, 2001, vol. 3, pp. 1886-1891.

[3] S. Krasser, H. L. Owen, J. Sokol, H.-P. Huth, and J. Grimminger, "Adaptive per-flow traffic engineering based on probe packet measurements," in Proc. 3rd Annual Communication Networks and Services Research Conf., 2005, pp. 131-136.

[4] V. Jacobson, "Congestion avoidance and control," in Proc. ACM SIGCOMM '88, Aug. 1998, pp. 314-329.

[5] S. Keshav, "A control-theoretic approach to flow control," in Proc. ACM SIGCOMM'91, Sept. 1993, pp. 3-15.

[6] R. L. Carter and M. E. Crovella, "Measuring bottle-neck link speed in packet-switched networks," in Performance Eval., vol. 27/28, pp. 297-318, Oct. 1996.

[7] C. Dovrolis, P. Ramanathan, and D. Moore, "Packet-dispersion techniques and a capacity-estimation methodology," IEEE/ACM Trans. Networking, vol. 12, pp. 963-977, Dec. 2004.

[8] M. Jain and C. Dovrolis, "End-to-end available bandwidth: Measurement methodology, dynamics, and relation with TCP throughput," IEEE/ACM Trans. Networking, vol. 11, pp. 537-549, Aug. 2003.

[9] K. Lai and M. Baker, "Measuring bandwidth," in Proc. INFOCOM'99, 1999, pp. 235-245.

[10] R. Kapoor, L.-J. Chen, M. Y. Sanadidi, and M. Gerla, "CapProbe: A simple and accurate capacity estimation technique," in Proc. ACM SIGCOMM'04, 2004, pp. 67-78.

[11] V. Paxson and S. Floyed, "Wide area traffic: the failure of Poisson modeling," IEEE/ACM Trans. Networking, vol. 3, pp. 226-244, Jun. 1995.

[12] A. Erramilli, O. Narayan, and W. Willinger, "Experimental queueing analysis with long-range dependent packet traffic," IEEE/ACM Trans. Networking, vol. 4, pp. 209-223, Apr. 1996

[13] H. S. Kim and N. S. Shroff "Loss probability calculations and asymptotic analysis for finite buffer multiplexers," IEEE/ACM Trans. Networking, vol. 9, pp.755-768, Dec. 2001.

[14] F.P. Kelly, "Notes on effective bandwidth," in Stochastic Networks: Theory and Applications, F.P. Kelly, S. Zachary, and I. Ziedins, Eds. Oxford, U.K.: Oxford Univ. Press, 1996, pp. 141-168.

[15] V. Paxson, "Measurements and analysis of the end-to-end Internet dynamics" Ph.D. dissertation, University of California, Berkeley, 1997.

[16] A. Pasztor and D. Veitch, "The packet size dependence of packet pair like methods," in Proc. IEEE/IEIP Int. Workshop Quality of Service (IWQoS), 2002, pp. 204-213.

[17] G. Jin, G. Yang, B. Crowley, and D. Agarwal, "Network characterization service (NCS)" in Proc. 10th IEEE symp. High Performance Distributed Computing, 2001, pp. 289-299.

[18] C. Dovrolis, P. Ramanathan, and D. Moore, "What do packet dispersion techniques measure?" in Proc. IEEE INFOCOM'01, 2001, pp. 905-914.

[19] B. Melander, M. Bjorkman, and P. Gunningberg, "A new end-to-end probing and analysis method for estimating bandwidth bottlenecks," in Proc. IEEE GLOBECOM, 2000, pp. 415-420.

[20] Cooperative Association for Internet Data Analysis, "NASA Ames Internet Exchange Packet Length Distributions," available at http://www.caida.org/analysis/AIX/plen_hist/.

[21] M. Kim "Internet application traffic monitoring and analysis," , $\mathrm{PhD}$ thesis, Pohang University of Science and Technology (POSTECH), Korea, 2004.

[22] C. Fraleigh et al., "Packet-level traffic measurements from the Sprint IP backbone," IEEE Network, vol. 17, pp. 6-16, Nov./Dec. 2003.

[23] D.J. Parish, K. Bharadia, A. Larkum, I.W. Phillips, and M.A. Oliver, "Using packet size distribution to identify real-time networked applications," IEE Proc.-Commun., vol. 150, pp. 221-227, August 2003.

[24] A. Leon-Garcia, Probability and Random Processes for Electrical Engineering, Addison Wesley Longman, Reading, MA, USA, 2nd edition, 1994.

[25] Y. Cheng, V. Ravindran, and A. Leon-Garcia, "Internet traffic characterization using packet-pair probing", submitted to IEEE INFOCOM 2007. 